\title{
OCTA Criteria in Orthognathic Surgery: A Review
}

Dr. Ch Shivakanth, MDS ${ }^{1 *}$, Dr. Metla Subbaiah Naidu ${ }^{2}$, Dr. Ratna Deepika Seshagiri, MDS ${ }^{3}$, Dr. Jacob John Plackal ${ }^{4}$, Dr. Naresh Vattikunta ${ }^{5}$, Dr. Sushil Bhagwan Mahajan ${ }^{6}$, Dr. Rahul Vinay Chandra Tiwari, FOGS, MDS ${ }^{7}$

${ }^{1}$ Consultant Oral and Maxillofacial Surgeon, Nizamabad Telangana, India

${ }^{2}$ Consultant Oral and Maxillofacial Surgeon and Implantologist, Indo American Dental Hospital, Yosufgoda Road, Opp State Home Ammerpet, Hyderabad Telangana, India

${ }^{3}$ Consultant Oral and Maxillofacial Surgeon, Vijayawada, Andhra Pradesh, India

${ }^{4}$ Consultant Oral and Maxillofacial surgeon, Believers Church Medical Centre, Konni, Pathanamthitta, Kerala, India

${ }^{5}$ Senior Lecturer, Department of Orthodontics, Dr's Sudha and Nageswara Rao Siddartha Institute of Dental Sciences, Chinnoutpally, Gannavaram, Krishna, Andhra Pradesh, India

${ }^{6}$ 2nd Yr Mds Orthodontics and Dentofacial Orthopedic, Dr. H.S.R.S.M. Dental College and Hospital Hingoli, Maharashtra, India

${ }^{7}$ Assistant Professor, Department of Oral and Maxillofacial Surgery, Sri Sai College of Dental Surgery, Vikarabad, India

DOI:10.36348/SJM.2019.v04i09.001

| Received: 13.08.2019| Accepted: 03.09.2019| Published: 15.09.2019

*Corresponding author: Dr. Ch Shivakanth

\section{Abstract}

Planning for conventional orthognathic surgery involves clinical examination, model analysis \& cephalometric analysis. Advances in the field of orthognathic surgery had prompted three-dimensional imaging and computer-assisted surgical planning of orthognathic procedures. However, even today clinical evaluation of face is considered gold standard in the diagnosis and treatment of orthognathic surgery. This paper throws light on the octa criteria in orthognathic surgery.

Keywords: Octa criteria, Orthognathic Surgery.

Copyright @ 2019: This is an open-access article distributed under the terms of the Creative Commons Attribution license which permits unrestricted use, distribution, and reproduction in any medium for non-commercial use (NonCommercial, or CC-BY-NC) provided the original author and source are credited.

\section{INTRODUCTION}

Orthognathic surgery is a surgical intervention that alters relationships of the jaws and dental arches. Facial esthetics is considered to be a critical factor in human interrelationship. Few patients have a unique constellation of dentofacial features that neither growth modification nor the camouflage would offer a possible solution. It requires a combined surgical and orthodontic procedure to correct major dentofacial deformities and malocclusion [1].

Orthognathic surgery is used to correct dentofacial deformities that cannot be treated with acceptable results by conventional orthodontics. The most frequently used surgical procedures for correcting the relationship of the jaws are Le Fort I osteotomy and sagittal ramus split osteotomy. Also often used are vertical ramus osteotomy, inverted L osteotomy, and variations and combinations of the above. ${ }^{2}$ Le Fort I was already described in 1867 and in 1953, Obwegeser introduced the sagittal split osteotomy [2, 3].

Various aspects of orthognathic surgery have been investigated, which has led to huge progress regarding efficient and predictable outcomes following surgical intervention. The most recent advances are three-dimensional imaging and computer-assisted surgical planning of orthognathic procedures. Conventionally, the diagnosis \& treatment planning of orthognathic surgery involved clinical examination, model analysis \& cephalometric analysis. Amongst these, even today clinical examination is considered to be the god standard in the diagnosis \& treatment planning in patients requiring orthognathic surgery.

\section{OCTA CRITERIA}

Octa criteria are a criterion adopted in orthognathic surgery patients for clinical evaluation. It includes the following.
1. Nasolabial angle
2. Lip protrusion
3. Facial proportion
4. Sub nasal perpendicular
5. Labiomental fold
6. Alar base
7. Nasal dorsum
8. Occlusal relationship

\section{Nasolabial Angle}

The nasolabial angle is formed by two lines, one from the nose, another from the upper lip, and both independent of each other. The angular measurement described by these two lines is a resultant of their individual inclinations [4]. The measurement of this 
angle alone provides inadequate information as it does not reveal which component is responsible for the variability. It could be the nose, the lip or both. Therefore, it is important to analyze each component of this angle to assist in the differential diagnosis of normal from its variation $[5,6]$.

The nasal dorsum, nasal tip \& columella play an important role in the formation of nasolabial angle. Acute nasolabial angle denotes protrusion of the upper anterior dentoalveolar component, increased upper incisor inclination or maxillary skeletal protrusion. An obtuse nasolabial angle denotes retrusion of the upper anterior dentoalveolar component, decreased upper incisor inclination or maxillary skeletal rettrusion.

Nasal analysis and quantitative data on the surgical movement and soft tissue changes need to be considered during the treatment planning process to predict soft tissue changes that can occur with dental and skeletal tissue alteration after surgery. Soft tissue changes are more predictable with mandibular surgery than maxillary advancement surgery due to soft tissue variability in midface region [7]. Evaluation of the nasolabial soft tissue is important when contemplating orthodontic treatment. Movement of the maxillary incisors in any of the three planes of space influences this area. However, consistent and reproducible methods of evaluating the nasolabial region are lacking [4].

\section{Lip Protrusion}

Upper lip inclination is an important keystone in treatment planning, both in orthodontics and in orthognathic surgeries. It is used to determine the anteroposterior lip position. Generally the lip upper lip should fall in such a way that $3 \mathrm{~mm}$ of upper incisors are exposed. Short upper lip is due to vertical deficiency and can lead to gummy smile \& hyperactivity of levator muscles. In presence of normal lip protrusion, excess of incisal exposure can occur in conditions of increased vertical height of the anterior maxilla and in deep bite cases.

The change in ULI in orthodontic treatment depends on the degree of retroclination/retraction of the maxillary incisors, the thickness and tonicity of the upper lip and the size of the space between the anterior dentoalveolus and the inner surface of the upper lip. ${ }^{8}$ The magnitude of the deviation of the upper lip inclination, whether it is due to the sagittal position of the maxilla, the inclination of the maxillary incisor teeth, the morphology and thickness of the overlying soft tissues of the upper lip, or any combination of the above, is an important factor in deciding whether orthodontic and/or orthognathic treatment may be required [8]. It is also an important factor in the perception of facial profile attractiveness and is the lower component of the nasolabial angle formation [9].

\section{Facial Proportion}

Pertaining to clinical evaluation of face with regards to facial proportions, individuals are encountered who exhibit imbalance between the vertical and horizontal components of the facial skeleton and other parts of the face. Some of these cases involve primary vertical dysplasia whilst in others vertical disproportion complicates horizontal and transverse abnormalities of growth [10].

The well balanced face can be divided in to three equal thirds. Vertical or anteroposterior abnormality can both affect the vertical balance of lips and chin in the lower third. These features will be altered by anteroposterior movement of the maxilla or mandible. Maxillary advancement brings the nasal tip forwards and tilts it upwards, and this effect is increased if the maxilla is simultaneously shortened vertically. Both advancement and shortening also widen alar flare and enhance the convexity of the para-alar regions. Shortening also widens the oral commissure, increasing lip vermillion exposure in centric occlusion of the teeth. Reverse effects follow facial lengthening by maxillary elongation. The alar flare is reduced, the lips are 'thinned', and the mouth narrows from side to side [10]. Treatment should always be planned to correct the anomalous marks of vertical disproportion and to avoid their accentuation.

\section{Subnasale Perpendicular}

A perpendicular line should be drawn perpendicular from the Frankfort Horizontal plane passing through the subnasale. It can be used for profile analysis. For an esthetic face the upper lip vermilion should fall on the perpendicular line drawn from the subnasale while the lower lip should be $2 \mathrm{~mm}$ and the soft tissue menton $4 \mathrm{~mm}$ behind the perpendicular plane. This line can also be used to identify dentofacial deformities in the sagittal plane.

\section{Mentolabial Fold}

Deep mentolabial fold is seen in patients with Skeletal Class II malocclusion while a shallow mentolabial fold is seen in patients with Skeletal Class III malocclusion. Deep mentolabial fold. Flared lower incisors, extruded upper incisors that roll out the lower lip, flaccid lower lip tone and abnormal morphology of lip itself are all factors that can affect the inclination of the lower lip and deepen the sulcus [11]. So uprighting the lower incisors, intruding the maxillary incisors and cheiloplasty to retract the lower lip all can help in reducing a deep sulcus [12].

\footnotetext{
Alar Base

In facial evaluation, it is to be understood that the length of the alar base should be equal to the intercanthal distance. All maxillary osteotomies performed for the correction of the dentofacial deformity will lead to altering of the alar base due to the decollation of the soft tissue surrounding the piriform
} 
aperture [13]. In order to minimalize this unwanted effect, a cinch suture in usually utilized along with the $\mathrm{V}-\mathrm{Y}$ suture in bimaxillary orthognathic surgery. Another option is to perform a pyriformplasty and anterior nasal spine recontouring.

\section{Nasal Dorsum}

An esthetic nose is evaluated for symmetry \& projection. In esthetic surgery, nasal evaluation has to be done pertaining to presence / absence of nasal hump, saddle nose, deviated nasal tip and asymmetry. These corrections can be carried out either by orthognathic surgery or rhinoplasty.

\section{Occlusal Relationship}

Evaluation of the face is incomplete without the evaluation of the dental component. Following the facial evaluation, the dental occlusal relationships have to be evaluated. Occlusion has to be checked for whether it is Class I, Class II or Class III, whetehr there is any cross-bite or deep bite and what is the Curve of Spee.

\section{CONCLUSION}

Inspite of the advances in the field of orthognathic surgery in the form of three-dimensional imaging and computer-assisted surgical planning of orthognathic procedures, clinical evaluation using the octa criteria is still considered to be the gold standard in the diagnosis and treatment planning of orthognathic surgery.

\section{REFERENCES}

1. Uppada, U. K., Sinha, R., Reddy, D. S., \& Paul, D. (2014). Soft tissue changes and its stability as a sequlae to mandibular advancement. Annals of maxillofacial surgery, 4(2), 132-137.

2. Larsen, M. K. (2017). Indications for orthognathic surgery-A review. Journal Oral Health Dent Manag, 16(2):1-13.

3. Steinhäuser, E. W. (1996). Historical development of orthognathic surgery. Journal of cranioMaxillofacial surgery, 24(4), 195-204.
4. Dua, V., Gupta, S., \& Singh, C. (2010). Evaluation of the nasolabial angle in the Indian population. Contemporary clinical dentistry, 1(2), 79-82.

5. Fitzgerald, J. P., Nanda, R. S., \& Currier, G. F. (1992). An evaluation of the nasolabial angle and the relative inclinations of the nose and upper lip. American Journal of Orthodontics and Dentofacial Orthopedics, 102(4), 328-334.

6. Lo, F. D., \& Hunter, W. S. (1982). Changes in nasolabial angle related to maxillary incisor retraction. American journal of orthodontics, 82(5), 384-391.

7. Nagori, H., \& Fattahi, T. (2017). Maxillary advancement surgery and nasolabial soft tissue changes. J Medical and Dental Sciences, 16, 2329.

8. Naini, F. B., Cobourne, M. T., McDonald, F., \& Wertheim, D. (2014). The aesthetic impact of upper lip inclination in orthodontics and orthognathic surgery. European journal of orthodontics, 37(1), 81-86.

9. Khamashta-Ledezma, L., \& Naini, F. B. (2014). Systematic review of changes in maxillary incisor exposure and upper lip position with Le Fort I type osteotomies with or without cinch sutures and/or VY closures. International journal of oral and maxillofacial surgery, 43(1), 46-61.

10. Henderson, D. (1981). The vertical dimensions in orthognathic surgery. British Journal of Oral Surgery, 19, 231-257.

11. Asif, S. M, Reddy, Y. M., Sreekanth, C., Reddy, B. V., Raj, G. K. P., \& Reddy, B. R. (2016). Evaluation of Soft Tissue Measurements in Various Skeletal Malocclusions of Kurnool Population- A Cephalometric Study. International Journal Oral Health Medical Research, 2(6):4144.

12. Legan, H. L., \& Burstone, C. J. (1980). Soft tissue cephalometric analysis for orthognathic surgery. Journal of oral surgery (American Dental Association: 1965), 38(10), 744-751.

13. Bita, R. (2011). Evaluation Of Alar Base In Patients With Surgical Maxillary Expamsion And Le Fort I Osteotomy. Med In Evol, 17(4):458-462. 